\title{
Ultraviolet and Visible State of the Molybdenum Oxide MoO3 thin Films Synthesized by Spray Pyrolysis Technique
}

\author{
H. H. Afify ${ }^{a}$, S. A. Hassana, A. Abouelsayed ${ }^{b}$, S. E. Demiana, \\ H. A. Zayed ${ }^{c}$
}

${ }^{a}$ Solid State Department, Physics Division, NRC, Dokki, Cairo 12622, Egypt

${ }^{b}$ Spectroscopy Department, Physics Division, NRC, Dokki, Giza 12622, Egypt

${ }^{c}$ Physics Department, Faculty of Girls for Art, Sciences and Education, Ain Shams University, Cairo, Egypt

Ultraviolet and Visible transmission measurements are performed over a wavelength range 190-2500 $\mathrm{nm}$ on the $\mathrm{MoO} 3$ thin films synthesized by spray pyrolysis technique at different substrate temperature and $3 \mathrm{~min}$. spray time. The appeared bands are fitted and deconvaluted by Lorentz model. The higher energy band corresponds to the transition from valance band to conduction band while the lower energy band corresponds to the transition between oxidation states of molybdenum. Two sub-bands are resolved which are affected by substrate temperature and corresponds the electron transition between the molybdenum oxidation states $\mathrm{Mo}^{4+}, \mathrm{Mo}^{5+}$ and $\mathrm{Mo}^{6+}$. The transition from $\mathrm{Mo}^{5+}$ to $\mathrm{Mo}^{6+}$ oxidation states show a blue shift up to $\mathrm{Tc}=325^{\circ} \mathrm{C}$. Above $\mathrm{Tc}$, the transition $\mathrm{Mo}^{5+}$ to $\mathrm{Mo}^{6+}$ increases more drastically, resulting in an anomaly in the temperature-induced shift at Tc. The anomaly can be attributed to the amorphous-to-crystalline phase transition at $325^{\circ} \mathrm{C}$. Also, both refractive index and extinction coefficient are calculated as a function of substrate temperature.

\section{Introduction}

Experimental studies have been made on the structural and optical properties of the molybdenum oxide. These studies have been motivated by scientific and technological interest [1,2]. They are a model system relatively easy in preparation by using many deposition techniques [3,4]. It has a great potential for applications including solar cell, flat panel displays, batteries, 
photo-detector, optical devices, sensors and electronics [5, 6]. For this reason, large efforts have been undertaken for reliable understanding of the basic properties of the molybdenum oxide nanostructure.

The electronic and optical properties of molybdenum oxide are closely correlated with their geometrical structure, namely their amorphous and crystalline structure, which results from using different substrate temperatures during the deposition process. Experimentally, structural and optical properties of amorphous and crystalline molybdenum oxide thin films have been investigated by Tarsame et al [7]. The author concluded that the as deposited $\mathrm{MoO} 3$ films by thermal evaporation under high vacuum are amorphous films got crystallized by increasing the annealing temperature up to $350^{\circ} \mathrm{C}$ [7]. He found that the XPS core level analysis shows the presence of three oxidation state in asdeposited films while there is only one oxidation state in the crystalline films. However, one can observe in his optical measurements two sub-band transitions in the crystalline films.

The aim of the present work is to investigate the effect of substrate temperature on the appeared absorption bands in the wavelength range from 200 up to $2500 \mathrm{~nm}$. These bands will be fitted and deconvoluted using Lorentz model. The spectral weight and energy of the arising sub-bands are correlated with the change in substrate temperature and compared with the data obtained from published XPS results on $\mathrm{MoO}_{3}$ thin film. Also, refractive index and extinction coefficient will be calculated.

\section{Experiment}

Several parameters are relevant to the spray pyrolysis system such as, distance between nozzle orifice and substrate surface, nozzle shape and dimensions, precursor material and concentration, spray time and substrate temperature, flow rates of gas and solution. Each of the above parameters is manipulated one at a time to produce films with reasonable adherence and homogeneity. It is found that the optimum parameters for the prime deposited films are: (i) solution flow rate $0.3 \mathrm{ml} / \mathrm{sec}$; (ii) filtered and dried compressed air $6 \mathrm{~N}$ cm-2 ; (iii) distance between nozzle orifice and substrate surface $30 \mathrm{~cm}$; (iv) precursor $\mathrm{MoCl} 5$; (v) solution molarity $0.2 \mathrm{M}$.

The used spray pyrolysis set-up to prepare $\mathrm{MoO} 3$ is described elsewhere [8]. The substrate on the heater surface and the spraying nozzle are settled in a glass enclosure to maintain temperature stability. The heater temperature is controlled to $\pm 1{ }^{\circ} \mathrm{C} \mathrm{Via} \mathrm{Cu} / \mathrm{Cu}$ constant thermocouple and control circuit. The glass substrate $(1.5 \times 2.0 \times 0.1 \mathrm{~cm})$ is cleansed chemically and ultra- sonically. 
The used spray pyrolysis set-up to prepare $\mathrm{MoO}_{3}$ is described elsewhere [8]. The substrate on the heater surface and the spraying nozzle are settled in a glass enclosure to maintain temperature stability. The heater temperature is controlled to $\pm 1^{\circ} \mathrm{C}$ Via $\mathrm{Cu} / \mathrm{Cu}$ constant thermocouple and control circuit. The glass substrate $(1.5 \times 2.0 \times 0.1 \mathrm{~cm})$ is cleansed chemically and ultra- sonically

The $0.2 \mathrm{M}$ spray solution is formed by dissolving Molybdenum pentachloride (Merck $99.6 \%$ purity) in bidistilled water. Filtered and dried compressed air is used as carrier gas. The above mentioned conditions are fixed at their optimum values. The prepared films are obtained by varying the substrate temperature from $200^{\circ} \mathrm{C}$ to $400^{\circ} \mathrm{C}$ at fixed spray time 3 minute. The Uv-Vis transmittance is measured at room temperature in the energy range $190-$ $2500 \mathrm{~nm}$ using a double beam spectrophotometer. The substrate is used as a reference through the measurements of $\mathrm{T}-\lambda$ for the samples.

\section{Results and Discussion}

The obtained $(T-\lambda)$ data are transferred to absorption $(\mathrm{A}-\lambda)$ by using Jasco program. The UV-Vis absorption spectra of the as deposited $\mathrm{MoO} 3$ thin films at various substrate temperature (a) $200^{\circ} \mathrm{C}$; (b) $250^{\circ} \mathrm{C}$; (c) $300^{\circ} \mathrm{C}$; (d) $325^{\circ} \mathrm{C}$; (e) $350^{\circ} \mathrm{C}$ and (f) $400^{\circ} \mathrm{C}$ at constant spray time 3 minutes are shown in Fig. (1). The absorption spectra of MoO3 film is described by using DrudeLorentz (DL) model [9]. It is reported that the absorption bands in transition metal oxide can be modeled by Gaussian or Lorentz profile. The MoO3 film is considered as being dense enough to obtain the optical features suitable to apply the Lorentzian model. Quantitative information about the effect of substrate temperature on the probable transitions is obtained by fitting the absorption spectra at each substrate temperature with Lorentz function.

$$
\epsilon(\omega)=\epsilon_{\infty}+\frac{4 \pi e^{2}}{m} \sum_{j} \frac{N_{j}}{\left(\omega_{j}^{2}-\omega^{2}\right)-i \gamma_{j} \omega}
$$

where $\gamma_{\mathrm{j}}$ and $\mathrm{N}_{\mathrm{j}}$ are the width and the density of electrons bound with the central frequency $\omega_{j}$, respectively. As a result of fitting, the spectrum is divided into two absorption bands appeared at different positions i.e. band 1 at $4.2 \mathrm{eV}$ and band 2 at $2.7-1.4 \mathrm{eV}$. Band 1 could be attributed to the energy gap corresponding to the transition from valence band to conduction band since it is sharp and no sub band. Band 2 is broad and deconvoluted to two sub-bands (E1 and E2) their position and intensity are significantly affected by substrate temperature. 

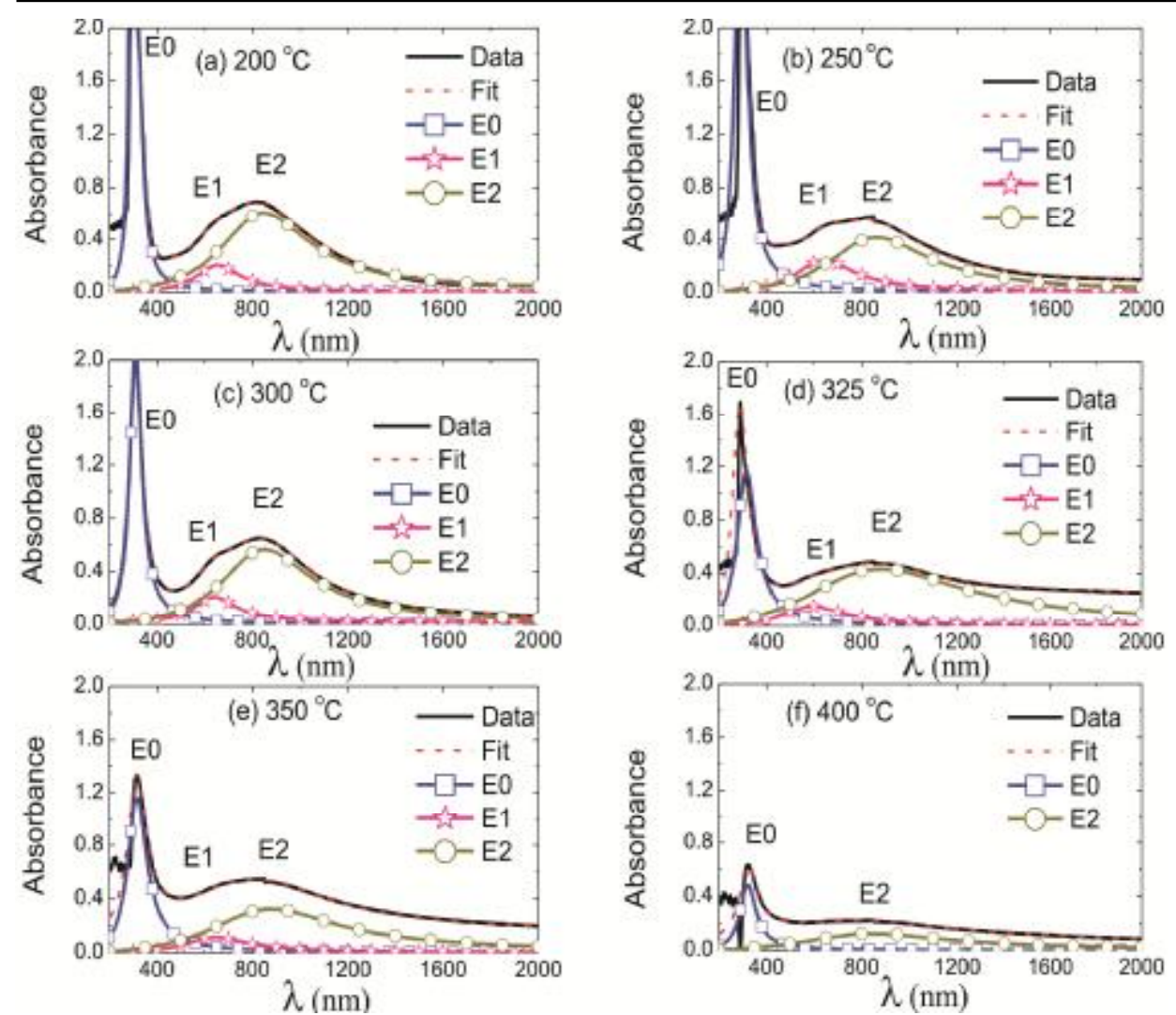

Fig. (1): Absorbance spectrum of the $\mathrm{MoO} 3$ thin films and the fit with Lorentz functions at different temperature: (a) $200^{\circ} \mathrm{C}$; (b) $250^{\circ} \mathrm{C}$; (c) $300^{\circ} \mathrm{C}$; (d) $325^{\circ} \mathrm{C}$; (e) $350^{\circ} \mathrm{C}$ and at (f) $400^{\circ} \mathrm{C}$ at spray time 3 minutes. The labels E0, $\mathrm{E} 1$ and $\mathrm{E} 2$ denote the band gab transition and the transitions between $\mathrm{Mo}^{5+}$ to $\mathrm{Mo}^{6+}$ states and $\mathrm{Mo}^{4+}$ to $\mathrm{Mo}^{5+}$ oxidation sates, respectively.

Band 2 with resolved sub-bands at each substrate temperature are shown in Fig. (2). Fig. 2 (a) shows absorbance spectra of the $\mathrm{MoO}_{3}$ film at $200^{\circ} \mathrm{C}$ together with the fitting curve and its components. We can clearly resolve two Lorentzian contributions E1 and E2 transitions at approximately $1.87 \mathrm{eV}$ and $1.4 \mathrm{eV}$ respectively. The two Lorentzian contributions E1 and E2 can be attributed to the electron transition from $\mathrm{Mo}^{5+}$ to $\mathrm{Mo}^{6+}$ states and from $\mathrm{Mo}^{4+}$ to $\mathrm{Mo}^{5+}$ oxidation sates, respectively as maintained by Tarsame et al [7]. 

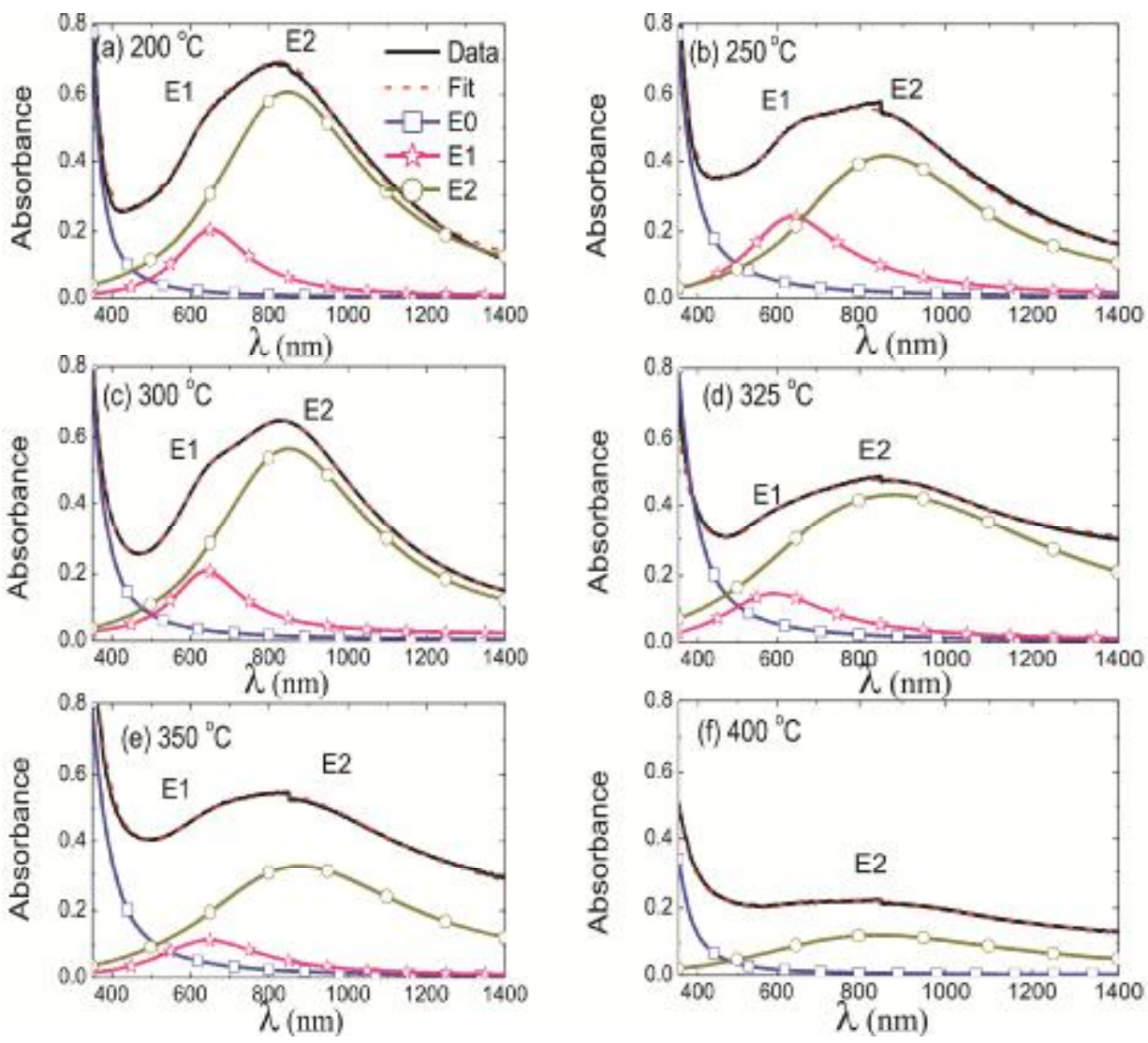

Fig (2): The fit with Lorentz functions of the $\mathrm{MoO} 3$ thin films at different temperature: (a) $200^{\circ} \mathrm{C}$; (b) $250^{\circ} \mathrm{C}$; (c) $300^{\circ} \mathrm{C}$; (d) $325^{\circ} \mathrm{C}$; (e) $350^{\circ} \mathrm{C}$ and at (f) $40^{\circ} \mathrm{C}$. The labels $\mathrm{E} 1$ and $\mathrm{E} 2$ denote the transitions between $\mathrm{Mo}^{5+}$ to $\mathrm{Mo}^{6+}$ states and $\mathrm{Mo}^{4+}$ to $\mathrm{Mo}^{5+}$ oxidation sates, respectively.

With increasing the deposition temperature the fine structure of the absorption bands are still observed in the spectrum up to $350^{\circ} \mathrm{C}$ (see Fig. 2 (a-e)). Thus, upon increasing temperature up to $350^{\circ} \mathrm{C}$ the absorption bands show slight decrease in intensity, and thus one can resolve two Lorentzian contribution for the broad absorption peak sub-bands nominated E1 and E2. With increasing the substrate deposition temperatures more up to $400^{\circ} \mathrm{C}$, the absorption peak is quite broad already, where E2 are centered at $1.42 \mathrm{eV}$ at $400^{\circ} \mathrm{C}$ (i.e. E1 is disappeared).

Such band (band 2 here) was observed [10] in $\mathrm{MoO}_{3}$ thin film photoinjected by hydrogen. This band was deconvoluted into three Gaussian 
peaks at 1.25-1.35, 1.75-1.95 and 2.25-2.75 ev. The relative contributions into the total absorption band are different for each sub-band. These bands were attributed to different color center namely $[11,12,13,14]$, the bulk paramagnetic center $\mathrm{Mo}^{5+}$, the surface paramagnetic center $\mathrm{Mo}^{5+}$ and the surface paired (diamagnetic center $\left[\mathrm{Mo}^{5+}, \mathrm{Mo}^{5+}\right]$ ). These observations are consonant with the work [15] here the paramagnetic centers in $\mathrm{Wo}_{3}$ and $\mathrm{MoO}_{3}$ were investigated. Here only two sub-bands are observed which may be related to the the paramagnetic center $\mathrm{Mo}^{5+}$. Also, the results are in a good agreement with the study of $\mathrm{MoO}_{3}$ films synthesized by thermal evaporation under high vacuum [7]. From the XPS measurements, the author concluded that there is $\mathrm{Mo}^{4+}$ to $\mathrm{Mo}^{5+}$ oxidation states in the amorphous state, while with increasing the annealing temperature there is only $\mathrm{Mo}^{6+}$ in the crystalline state. The origin of these oxidation sates in the amorphous films result from the oxygen vacancies in the films in which the vacancies are created during deposition of $\mathrm{MoO}_{3}$ films [7]. Deb et al [16] suggested an explanation of the formation of such oxidation sates $\mathrm{Mo}^{4+}$ to $\mathrm{Mo}^{5+}$ in the amorphous films as results of the electrons trapping in the oxy- gen vacancies. Here, by increasing the deposition temperature, the oxidation states are reduced by removing the major fraction of oxygen vacancies.

The obtained Lorentz functions describing the absorption features of the $\mathrm{MoO}_{3}$ film sub-bands $\mathrm{E} 1$ and E2 are plotted in Fig. (3a) as a function of temperature. The most important results are the following: with increasing the deposition temperature, the energies of E1 show slight blue shift up to $\mathrm{Tc}=$ $350^{\circ} \mathrm{C}$, while those for E2 are nearly stable significant. Above Tc, the energies of the E1 increase with increasing the substrate temperature, resulting in an anomaly in the temperature-induced shift at Tc. The anomaly can be attributed to the amorphous-to- crystalline phase transition at $350^{\circ} \mathrm{C}$. At $400^{\circ} \mathrm{C}$, the E1 cannot be distinguish from $\mathrm{E} 2$ suggesting that the $\mathrm{Mo}^{5+}$ oxidation sates are mixed with the $\mathrm{Mo}^{4+}$ states at this temperature to produce only one broad band centered around $1.4 \mathrm{eV}$ (see Fig. 2 f).

There is no change in the band position of E2 transition. The spectral weights of the E1 and E2 absorption bands as a function substrate temperature is shown in Fig. (3 b). With increasing the substrate temperature the spectral weights is decreased indicating that the density of the oxidation states $\left(\mathrm{Mo}^{4+}\right.$ to $\left.\mathrm{Mo}^{5+}\right)$ are reduced. 

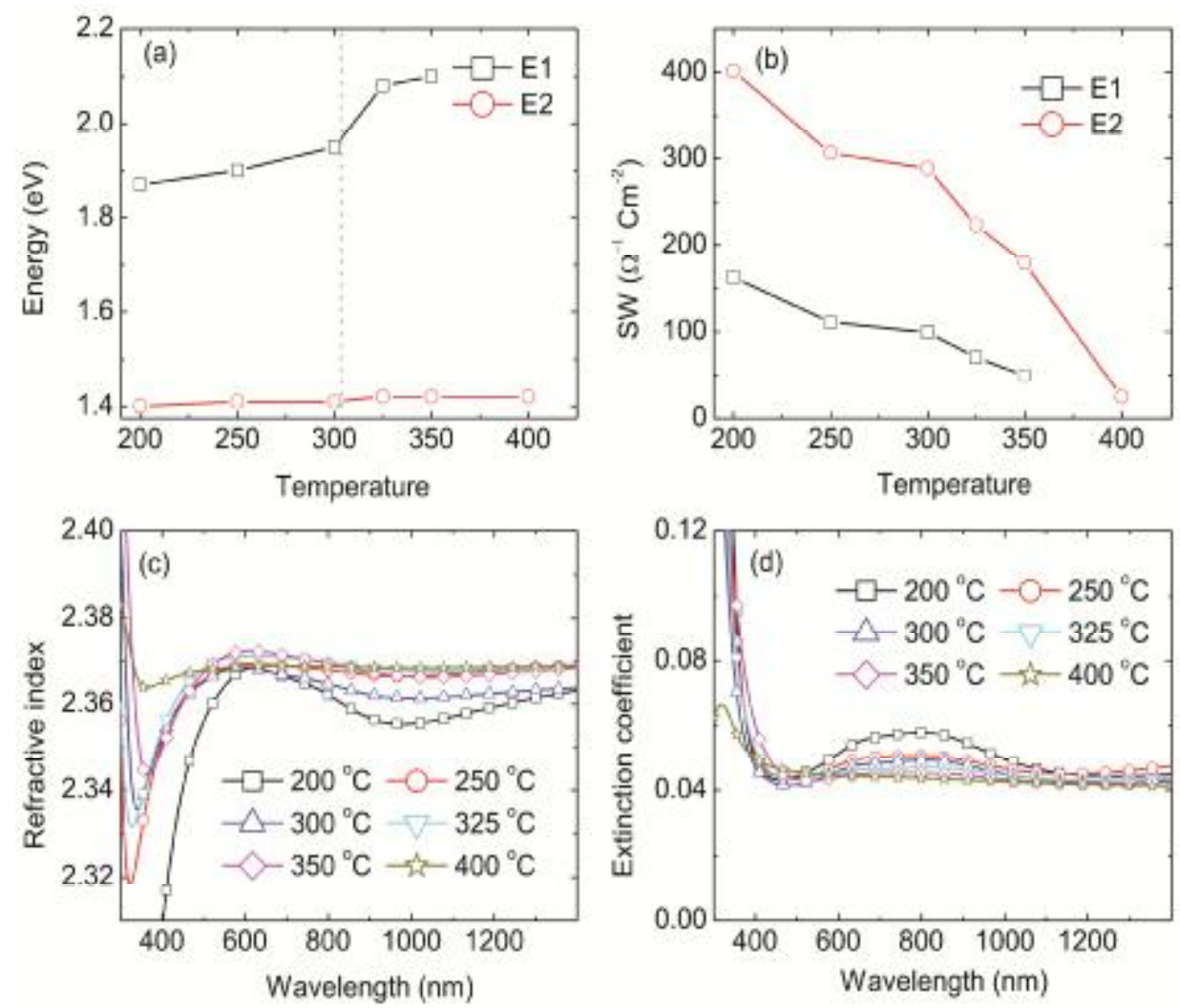

Fig. (3a): Temperature dependence of the transition energies of sub-band E1 and E2 resolved from band at longer wavelength. The vertical dashed line marks the critical temperature of the structural phase transition. (b) The spectral weights of the E1 and E2 absorption bands as a function of temperature. The calculated (c) refractive index $\mathrm{n}(\omega)$ and (d) Extinction coefficient $\mathrm{k}(\omega)$ obtained by fitting the absorption spectrum.

A considerable number of studies have also been made on the effect of substrate temperatures on the optical properties of the $\mathrm{MoO}_{3}$ thin films $[17,18]$. It was found that the band gap of the $\mathrm{MoO} 3$ shifts toward higherenergy regions as the oxygen vacancies of the sample is increased [17]. In addition, the energies of the absorption bands associated with the $\mathrm{MoO}_{3}$ oxidation states decreases as the oxygen content of the material rises, where the samples prepared with low substrate temperature indicate the rise of oxygen content of the samples. On the other hand, the oxygen vacancies are decreased with increasing the substrate deposition temperature and this causes the $\mathrm{MoO}_{3}$ to become basically crystalline $[17,18]$. 
To obtain the refractive index $n(\omega)$ (real part of the complex refractive index) we have fitted the absorption spectrum with Lorenz model. This has been achieved by first modeling the refractive index $\hat{n}=n+i k$ as follows:

$$
\begin{aligned}
& \epsilon_{\mathrm{l}}(\omega)=n^{2}-k^{2}=\epsilon_{\omega}+\sum_{j} \frac{\left.T_{j}^{2}(\omega)_{j}^{2}-\omega^{2}\right)}{\left.(\omega)_{j}^{2}-\left(\omega^{2}\right)+\gamma_{j}^{2} \omega\right)^{2}} \\
& \epsilon_{2}(\omega)=2 n k=\sum_{j} \frac{T_{j}^{2} \gamma_{j} \omega}{\left.\left.(\omega)_{j}^{2}-\omega\right)^{2}\right)+\gamma_{j}^{2}\left(\omega^{2}\right.}
\end{aligned}
$$

where $\gamma_{j}, T j$ and $\omega j$ are the width, the oscillator strength, and the central frequency, respectively. No significant changes have been observed in $\mathrm{n}$ at higher wavelengths with changing the de- position temperature shown in (Fig. 3 c). Below $600 \mathrm{~nm}$ the refractive index varies sharply near the band edge. The extinction coefficient $\mathrm{K}$ values at all deposition temperature demonstrated a peak between 2.7 and $1.4 \mathrm{eV}$ (see Fig. 3 (d)). This is in agreement with the interpretation of recent measurements on $\mathrm{MoO}_{3}$ under temperature, pointing out the weak change of the refractive index at higher wavelength i.e. higher than 600 $\mathrm{nm}[19]$.

\section{Summary}

The absorption spectra for as deposited $\mathrm{MoO}_{3}$ thin film de posited by spray pyrolysis at different substrate temperature and constant spray time 3 minutes is analyzed. The obtained spectra are fitted by Lorentz model. An appeared absorption band at high energy side corresponds to the energy gap while the other appeared at low energy side corresponds to the oxidation state transitions. As a result of deconvaluted two sub- bands are produced their position and intensity are reasonably changed by substrate temperature. Thus interpreted by the change in the population of the oxidation states $\mathrm{Mo}^{4+}, \mathrm{Mo}^{5+}$ and $\mathrm{Mo}^{6+}$. There is an anomaly at around $\mathrm{Tc}=325^{\circ} \mathrm{C}$ this may be due to crystallization of the sample. The refractive index and extinction coefficient are varied sharply near the absorption band edge, while slight change is observed in the long wavelength range. 


\section{References}

1. O.M. Hussain, K.S. Rao, Mater Chem and Phys, 80, 646 (2003).

2. E.M. Gaigneaux, K.I. Fukui, Y. Iwasawa, Thin Solid Films, 374, 58 (2000).

3. M. Kharrazi, A. Azens, L. Kullman, C.G. Granqvist, Thin Solid Films, 295, 121 (1997).

4. N. Miyata, T. Suzuki, R. Ohyama, Thin Solid Films, 281, 222 (1996).

5. A.M, Taurino, A. Forleo, L. Francioso, P. Siciliano, M. Stalder, Nesper R. Appl Phys Lett, 88, 152113 (2006).

6. Z.H. Wen, Q. Wang, J.H. Li. J Nanosci Nanotechnol, 6, 2122 (2006)

7. T.S. Tarsame Sian, G.B. Reddy. Solar energy materials and solar cells, 82, 386 (2004).

8. H.H. Afify, S.A. Mahmoud, A. Ashour, Thin Solid Films, 263, 251 (1995).

9. M. Dressel, G.Grner, Electrodynamics of Solids (Cambridge University press, UK, (2002).

10. A. Gavrilyuk, U. Tritthart, W. Gey, Solar Energy Materials and Solar Cells, 95, 1851 (2011).

11. U. Tritthart, A. Gavrilyuk, W. Gey. Solid State Commun, 105, 657 (1998).

12. U. Tritthart, W. Gey, A.I. Gavrilyuk, Ionics, 4, 308 (1998).

13. U. Tritthart, W. Gey, A.I. Gavrilyuk, Electrochim Acta, 44, 3049 (1999).

14. A.I. Gavrilyuk, G. Borstel, A. Krumins, D. Millers, NATO science series, 3. High Technology Kluwer Academic Publishers, 77, 438 (2000).

15. R. Gazzinelli, O.F. Schirmer, J Phys C: Solid State Phys, 10L, L149 (1977).

16. S.K. Deb, J.A. Chopoorian. J Appl Phys, 37, 4825 (1966).

17. R. Cardenas, J. Torres, J.E.Alfonso, Thin Solid Films, 478. 151 (2005).

18. M. Dhanasankar, K.K. Purushothaman, G. Muralidharan, Solid State Sciences, 12, 251 (2010).

19. M.F. Al-Kuhaili, S.M.A. Durrani, I.A. Bakhtiari, A.M. Al-Shukri, Optics Communications, 283, 2862 (2010). 